\title{
Multilinguales
}

Pratiques littéraires, linguistiques, pédagogiques, didactiques et médiations culturelles contemporaines

\section{Compétence interculturelle et enseignement des langues maternelles en Afrique francophone contemporaine}

Intercultural Competence and Teaching Mother Tongues in Contemporary

Francophone Africa

\section{Christiane Mouto Betoko}

\section{(2) OpenEdition}

1 Journals

\section{Édition électronique}

URL : https://journals.openedition.org/multilinguales/3163

DOI : $10.4000 /$ multilinguales.3163

ISSN : 2335-1853

Éditeur

Université Abderrahmane Mira - Bejaia

\section{Édition imprimée}

Date de publication : 1 juin 2013

Pagination : 95-104

ISSN : 2335-1535

\section{Référence électronique}

Christiane Mouto Betoko, «Compétence interculturelle et enseignement des langues maternelles en Afrique francophone contemporaine », Multilinguales [En ligne], 1 | 2013, mis en ligne le 01 juin 2013, consulté le 01 juillet 2021. URL : http://journals.openedition.org/multilinguales/3163 ; DOI : https:// doi.org/10.4000/multilinguales.3163

Ce document a été généré automatiquement le 1 juillet 2021.

\section{(c)}

Multilinguales est mise à disposition selon les termes de la Licence Creative Commons Attribution Pas d'Utilisation Commerciale - Pas de Modification 4.0 International 


\title{
Compétence interculturelle et enseignement des langues maternelles en Afrique francophone contemporaine
}

\author{
Intercultural Competence and Teaching Mother Tongues in Contemporary \\ Francophone Africa
}

Christiane Mouto Betoko

1 Le Conseil de l'Europe, en préconisant l'approche interculturelle dans l'enseignement des langues, plonge l'enseignement/apprentissage des langues dans une ère nouvelle, dans laquelle enseignants et apprenants interrogent sans cesse les rapports qu'ils entretiennent d'une part, avec leur culture et d'autre part, avec la culture de ceux avec qui ils partagent la même langue (dans le cas des langues maternelles et secondes) ou encore de ceux dont ils apprennent la langue (dans le cas des langues étrangères). Cette nouvelle donne crée à la fois un retour aux sources et un pas vers l'altérité, alors qu'en Europe la didactique des langues et cultures semble avoir pour objectif majeur, l'acquisition de la compétence interculturelle par les différents publics évoluant en milieu institutionnel. En Afrique francophone, il faut d'abord instaurer un débat autour de l'enseignement/apprentissage des langues maternelles qui occupent une place importante dans la vie socioculturelle. Nous pensons que la prise en compte de la langue maternelle à l'école est un prérequis dans la formation à l'interculturel, car, en Afrique francophone en général, les apprenants débutent leur scolarité dans une langue différente de celle(s) qu'ils utilisent en famille ou en communauté.

$2 \mathrm{Au}$ regard de la place accordée, aujourd'hui, à la culture dans l'enseignement/ apprentissage des langues, il est indispensable de s'interroger sur celle des langues maternelles et de la culture africaine à l'école. Pour tenter d'y répondre, nous allons nous appuyer sur la notion de compétence interculturelle. 


\section{Les langues maternelles en Afrique francophone}

3 L'importance de l'enseignement en langue maternelle, à travers les différents résultats obtenus au cours de multiples expériences menées par des didacticiens d'horizons et de cultures divers, n'est plus à démontrer aujourd'hui : « [la langue maternelle] détermine le développement affectif, cognitif, social de chaque individu, conséquemment ses motivations et ses capacités à apprendre d'autres langues par la suite " (Defays, 2003 : 28). Ainsi conçues, les langues maternelles peuvent constituer un atout pour la formation à l'interculturel :

[L'enseignant des langues est alors capable] de faire saisir à ses élèves la relation entre leur propre culture et d'autres cultures, de susciter chez eux un intérêt et une curiosité pour "l'altérité ", et de les amener à prendre conscience de la manière dont d'autres peuples ou individus les perçoivent - eux-mêmes et leur culture. (M. Byram et al., 2002 : 10-11)

4 Ces apprenants acquièrent leur propre culture dans leur quotidien, à travers les échanges en langue maternelle, à l'école et hors de la salle de classe. Dans les pays d'Afrique francophone, les langues maternelles sont non seulement reconnues par les différents États de cette partie de l'Afrique mais elles font également partie intégrante des différentes politiques linguistiques adoptées par ces États : «Elle [La République du Cameroun] œuvre pour la protection et la promotion des langues nationales» (Paul Biya, 1996) comme en témoigne le texte de loi $n^{\circ}$ 98/004 du 14 avril 1998 portant orientation de l'éducation au Cameroun qui stipule que » l'éducation a pour objectif: [...] la promotion des langues nationales » (Jacques Leclerc, 2011).

5 L'identité culturelle de ces apprenants d'Afrique francophone se caractérise par la reconnaissance des langues nationales qui doivent être enseignées et apprises en milieu institutionnel. En d'autres termes, l'intégration des langues nationales au sein de l'école fait de l'apprenant un individu conscient de son appartenance à une entité culturelle : "L'apprenant doit être en mesure d'affirmer sa propre identité tout en acceptant et comprenant les différences qui existeraient avec celle de l'autre et de saisir la complexité qui réside dans la relativité existant entre les différents systèmes culturels » (Samira Boubakour, $2010: 18)$.

6 Les besoins de l'apprenant en terme de formation à la culture maternelle propre sont certes identifiés mais, ne sont pas intégrés à leur cursus de formation. Certains écrivains parlent même de "la mort programmée des langues autochtones" (Guimatsia, 2012). L'ancrage de la langue française à tous les niveaux de l'éducation contribue à la mise en difficulté de ces apprenants qui vivent dans des milieux sociaux multilingues au sein desquels se mêlent langue(s) autochtone(s) du père et de la mère, langue véhiculaire utilisée dans la région, parlers composites (pidgin english ou camfranglais) et enfin le français langue officielle. L'influence de ces facteurs sur la scolarisation des apprenants a conduit à l'adoption, par les linguistes, d'une approche qui vise l'introduction des langues maternelles aux côtés de la langue française :

Il s'agit dans le contexte multilingue qui est le nôtre [le contexte camerounais] et conformément aux résolutions de Libreville en 2003, de prendre en compte les langues africaines à tous les niveaux de l'enseignement de la langue française pour un meilleur enracinement dans le terroir et un établissement des différences et des complémentarités. (Nola, $2006: 128)$

7 On aboutit ainsi aux projets relatifs à l'enseignement bilingue que l'Organisation internationale de la Francophonie (OIF) a instauré dans certains pays africains où le 
français est langue officielle sans pourtant être langue maternelle. Il s'agit de la zone arabophone de l'Afrique (le Maroc, la Tunisie et le Liban) et de l'Afrique subsaharienne francophone (le Burkina Faso, la Guinée, le Mali, le Niger, la République démocratique du Congo).

D'après le rapport de l'OIF, ces actions ont eu pour résultats la production d'outils pédagogiques :

Des guides d'apprentissage du français en didactique convergente ont été produits en fonction du zonage et des langues. Ainsi, huit bi-grammaires français/langues nationales ont été produites pour l'Afrique subsaharienne dans les langues transfrontalières véhiculaires du continent que sont le mandingue, le fulfulde, le haoussa, le swahili, le songhay-zarma-dendi, le wolof et le moré. [...]. Ces différents guides sont en phase d'expérimentation sur le terrain pour mieux prendre en compte les besoins des enseignants. Ils sont suivis et évalués dans chaque pays par un comité de pilotage créé auprès du ministère de l'Éducation. (Wolff et Gonthier, 2010)

9 Par ailleurs, il ressort de ce même rapport de l'OIF, que les expériences portant sur la pédagogie convergente, en 1979 au Mali, n'ont abouti à rien de concluant du fait de l'absence de réflexions pédagogiques et didactiques sur les questions de l'articulation et de la convergence des langues maternelles et du français. Pour tenter d'apporter une solution à ce problème, de nouveaux projets voient le jour. Il s'agit par exemple du projet ELAN-Afrique (École et langues nationales en Afrique), qui est un prolongement de l'enquête de terrain menée en 2007 sous la dénomination LASCOLAF (Langues de scolarisation dans l'enseignement fondamental en Afrique subsaharienne francophone). C'est un projet de trois ans, à compter de l'année 2012, axé sur le développement de l'enseignement bilingue français/langues partenaires dans huit pays d'Afrique subsaharienne :

Cette nouvelle initiative née d'un partenariat entre l'OIF, maitre d'œuvre du projet, l'Agence universitaire de la Francophonie (AUF), l'Agence française de Développement (AFD) et le Ministère français des Affaires étrangères et européennes, vise à accompagner les pays d'Afrique sub-saharienne francophones, à développer une éducation au primaire de qualité et à faciliter l'implantation d'un enseignement bilingue alliant langues nationales et français pour tous les enfants et en particulier ceux des zones rurales pour remédier à l'échec scolaire dû à la difficulté d'acquisition de la langue française. [...]. ÉLAN-Afrique propose de soutenir les plans d'actions des pays qui souhaitent étendre progressivement l'enseignement en langues nationales dans les systèmes éducatifs au niveau primaire. Huit pays francophones (Bénin, Burkina Faso, Burundi, Cameroun, Mali, Niger, République démocratique du Congo et Sénégal) ont adhéré à ce projet, certains pour commencer les expérimentations, d'autres pour les consolider. (Organisation Internationale de la Francophonie, 2011)

10 Ces projets sont conçus pour améliorer la formation des enfants africains. Il existe également des programmes de formation d'enseignants de langues et cultures africaines. En 2009-2010, il a été créé, à l'École Normale Supérieure de Yaoundé, au Cameroun, la filière "Langues et cultures camerounaises "(LCC) qui a pour objectif pour la formation à l'interculturel en adéquation avec les langues et cultures du Cameroun. 


\section{La langue française et la culture africaine francophone}

11 Alors que Jean-Louis Chiss (2006: 11-16) porte ses espoirs sur les relations tissées entre l'enseignement des langues et l'anthropologie culturelle pour donner une nouvelle impulsion aux études des textes littéraires, l'Afrique francophone voit dans ces relations, une opportunité pour introduire ses cultures en recourant au texte littéraire. Ces textes africains permettent ainsi aux enseignants francophones, en Afrique, de pratiquer un enseignement de la culture littéraire et artistique africaine dont Luc Collès (2006) décrit les trois nouveaux enjeux majeurs selon la nouvelle donne scolaire :

un enjeu psychologique: la nécessité de transmettre aux jeunes générations des repères identitaires collectifs à travers un patrimoine littéraire commun, et plus spécifiquement celui qui s'enracine dans la langue maternelle et dans la culture nationale ;

un enjeu pratique: la nécessité de transmettre un bagage littéraire suffisant pour pouvoir décoder les nombreuses allusions, parodies et réécritures en tous genres qui se fondent sur les grandes références de la littérature ;

un enjeu proprement didactique : la nécessité de transmettre les grandes références (courants et mouvements, textes et auteurs prototypiques) qui permettent de comprendre les étapes et le sens de l'évolution du fait littéraire (18).

D'une part, l'enjeu didactique caractérise la dimension informative de cette approche et d'autre part, les enjeux psychologique et pratique renvoient à l'analyse des faits culturels autochtones et d'ailleurs.

Ces différents enjeux contribuent à la formation à l'interculturel qui implique l'enseignement de la langue maternelle et des langues seconde et étrangère. On peut relever à ce sujet que les écrivains d'Afrique francophone, par le biais de leurs œuvres, ont permis de transmettre les cultures et les valeurs africaines par le biais de la langue française. Nombreux sont les textes d'écrivains francophones qui embrassent les enjeux psychologiques, pratique et didactique, dont l'usage ne connaît pas de frontières en Afrique francophone.

14 Cependant, on relève depuis quelques années, dans certains pays de cet espace francophone, une préférence pour les écrivains du terroir. On peut citer à ce sujet quelques œuvres littéraires inscrites au programme de l'enseignement secondaire général au Cameroun de l'année scolaire 2010-2011 (Louis Bapes-Bapes, 2010).

Alors que dans les années 90, parmi les textes littéraires destinés aux apprenants des classes de $6^{\text {ème }}$ et de $5^{\text {ème }}$ (première et deuxième années du collège respectivement), on retrouvait en tête de liste Les contes d'Amadou Koumba du sénégalais Birago Diop, aujourd'hui, les ouvrages des écrivains camerounais à l'instar d'Anya Noah (Les chants de la forêt) et de Patrice Kayo (Fable des montagnes) occupent la rubrique réservée aux livres de contes africains. Toujours est-il que l'objectif est resté le même: ces récits mettent en scène des personnages, des animaux de la région ainsi que des éléments naturels dans le but de décrire la vie traditionnelle et de délivrer des leçons de sagesse. Ce sont les contes et légendes, récits mythiques et imaginaires, proverbes et devinettes, de la tradition orale des cultures africaines.

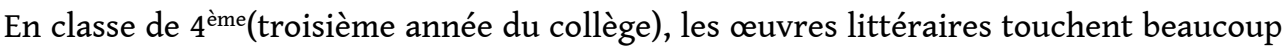
plus à la vie en société. Ce sont des pièces de théâtre (La forêt illuminée de Gervais Mendo ZE, Trois prétendants un mari de Guillaume Oyono Mbia), des nouvelles (Les 
Bimanes de Severin Cécile Abéga, Les vins aigres de Gabriel Kuitche) ou encore des récits merveilleux (Grain de poussière de Guillaume Nana). Dans cette société où les parents marient leur fille au "plus offrant " (Trois prétendants un mari), où des hommes et des femmes fidèles à la religion chrétienne pratiquent tout de même le culte des ancêtres (Les vins aigres), on peut encore tirer des leçons des « bimanes », villageois ou citadins.

Les œuvres de la classe de $3^{\text {ème }}$ (quatrième année du collège) interpellent les apprenants pour leur faire prendre conscience de certains faits de société. Le roman Africa Ba'a de Medou Mvomo met en exergue l'opposition ville/village, modernité/tradition pour les insérer dans un schéma différent où l'une et l'autre se complètent pour construire l'homme idéal de demain, des hommes forts de leur langue et culture ancestrales, ayant appris à vivre avec leur tradition dans la modernité, pour une meilleure intégration dans le village planétaire. Le roman Petit Jo, enfant des rues d'Evelyne Mpoudi Ngollénarre décrit la vie des enfants pauvres qui errent dans les rues des grandes villes camerounaises et dont les passants en ignorent le quotidien difficile.

Toutes ces œuvres littéraires sont compatibles avec les trois enjeux de Collès, bien que de façon limitée. C'est le cas de la perspective psychologique de cet enseignement car, les textes ne sont pas écrits dans les langues maternelles des apprenants, véhicules des faits culturels. En dépit de ces barrières, on ne peut ignorer le rôle de ces textes dans la transmission des us et coutumes de l'Afrique francophone.

Ces textes littéraires écrits en français et sur lesquels reposent les activités de lecture et de production écrite représentent alors les seuls garants de la culture africaine en classe de langue, même s'il ne s'agit que de cours de langue seconde. Apprendre sa langue et sa culture maternelles par l'intermédiaire d'un cours de langue seconde apparaît comme un exercice difficile. À ce propos, Andrée-Marie Diagne (2006) déclare : «Si la littérature francophone doit faire vivre et faire connaître les traditions des peuples en contact, l'une des voies de transmission des cultures dans l'espace francophone est la maîtrise par l'écrivain de sa culture et de sa langue maternelle » (26).

20 Ainsi l'écrivain d'aujourd'hui, initié à la culture orale traditionnelle dans sa langue maternelle transmet ce patrimoine aux jeunes générations, dans une autre langue. Comme le disait Sa'ah François Guimatsia (2012), à propos des Camerounais, dans un article relatif au bilinguisme officiel: "Ceux-ci ne devraient pas se présenter au rendezvous "du donner et du recevoir" en quémandeurs complexés, mais en contributeurs éclairés à la culture universelle ».

21 Ces propos s'adressent également à tous les pays d'Afrique francophone effectivement participants et non observateurs de la mouvance interculturelle qui réside dans le retour des langues maternelles à l'école.

\section{La compétence interculturelle à l'école d'aujourd'hui}

Vincent Louis, professeur de littérature et de didactique du Français langue étrangère, principaux domaines de ses nombreuses recherches, fait un inventaire des différentes méthodes d'enseignement/apprentissage développées sur la base de la compétence interculturelle, approche indispensable au processus d'acquisition des langues à l'école (Louis, 2007). 
23 En effet, son ouvrage a pour objectif principal la mise en œuvre d'activités pédagogiques nécessaires à l'acquisition de la culture par le biais d'interactions verbales. Pour ce faire, il définit au préalable les concepts de " compétence culturelle » et de "découverte interculturelle», en mettant en exergue les rapports qu'elles entretiennent dans leur contribution à la compétence de communication.

24 Pour la méthodologie qu'il prône, elle suit les quatre options de l'approche interculturelle: "l'approche linguistique de l'interculturel», «l'approche de l'interculturel par les stéréotypes », « l'approche anthropologique de l'interculturel » et « l'approche de l'interculturel par l'ethnographie de la communication ».

Si Louis propose une analyse de la « compétence (inter)culturelle », dans le cadre de la didactique du français langue étrangère (FLE), cela n'exclut cependant pas une analyse relative à l'enseignement/apprentissage de la langue maternelle à partir des mêmes approches car, comme le signale Collès (2006: 17) : «Une composante essentielle du territoire partagé entre les différentes didactiques du français consiste dans le rapport étroit qui unit la langue et la culture», d'où la notion de «langue-culture » couramment employée à l'heure actuelle. En d'autres termes, les didactiques des langues maternelles, secondes et étrangères peuvent faire l'objet des mêmes approches pour leur enseignement au niveau de la langue et de la culture.

26 L'approche linguistique de l'interculturel est la première méthode examinée par Louis. Cette approche est caractérisée par deux principales hypothèses: l'hypothèse lexicologique de Galisson et l'hypothèse de l'implicite de Collès (cf. Louis, 2007 : 131-136).

27 D'après ces théoriciens, «tous les membres d'une communauté ont une culture quotidienne transversale en commun » (Louis, $2007: 131$ ) que l'on retrouve dans les « mots à charge culturelle partagée » du point de vue de Galisson, ou encore dans « le savoir partagé, les tropes, les connotations, les allusions et les traits d'esprit » du point de vue de Collès. Toutes ces notions font références aux valeurs des implicites qui existent dans chacune de nos cultures et que nous partageons dans nos différents échanges au quotidien, avec les membres de nos différentes communautés, par le canal de notre langue première, la langue maternelle.

28 La seconde méthode porte sur l'approche de l'interculturel par les stéréotypes, approche essentiellement dominée par les recherches de Geneviève Zarate : „Éduquer à la perception interculturelle sans recourir au traitement des stéréotypes et à l'analyse critique des documents [...] que propose Zarate ne semble désormais plus possible » (Louis, 2007 : 141). Selon Zarate, les réalités culturelles présentes dans les documents authentiques ne sont rien d'autres que des représentations de la culture bâties sur la connaissance du monde par un groupe social. Cette approche met en exergue la place des représentations dans la relation culture maternelle - culture cible. Le sujet apprenant est appelé à se remettre en question en s'appuyant sur l'image qu'il a de luimême, avant d'envisager le repérage des stéréotypes qui existent chez l'autre à partir de l'analyse des documents authentiques dont la pertinence sociologique doit être évaluée.

29 Se pose alors la question de l'apprenant africain francophone dont la scolarisation se fait à travers la langue française : peut-il avoir une image de lui-même qui lui est propre, c'est-à-dire qui repose sur son identité culturelle? Les représentations qu'il a de sa culture sont-elles fondées? 
Louis analyse l'approche anthropologique de l'interculturel en s'appuyant sur l'hypothèse littéraire de Collès d'une part, et sur l'hypothèse de l'anthropologie comme herméneutique développée par Porcher et Abdallah-Pretceille, d'autre part :

Les méthodes anthropologiques devraient permettre aux apprenants d'apprendre à interpréter les éléments culturels présents dans tout acte de communication [...] [dans le but] de voir comment chaque locuteur utilise des faits de sa culture pour DIRE et SE DIRE, pour agir et s'affirmer. (Louis, $2007: 142$ )

31 Le texte littéraire est considéré ici comme le lieu de rencontre des cultures. L'apprenant doit s'y plonger avec des objectifs bien précis, pour découvrir comment l'étranger se manifeste dans sa culture à partir du langage qu'il emploie. L'apprenant francophone saura-t-il repérer les éléments pragmatiques nécessaires au dialogue auquel il est invité, dans ce lieu de rencontre qu'est le texte littéraire ? L'établissement des liens avec sa culture sera encore confronté à son ignorance des textes de sa langue maternelle.

32 Si nous nous en tenons aux trois enjeux de la didactique des langues évoqués plus haut, nous aboutissons à la conclusion selon laquelle l'absence de culture littéraire en langue maternelle pourrait être la cause principale des difficultés que connaissent les apprenants francophones dans les exercices de production écrite. La langue maternelle a donc une place indéniable dans l'acquisition de la compétence interculturelle en Afrique francophone, comme ailleurs.

33 La dernière méthode de Vincent Louis est celle de l'approche de l'interculturel par l'ethnographie de la communication dont le développement repose sur une combinaison de théories ethnographiques et de concepts propres à la didactique du FLE. Le processus d'acquisition de la compétence interculturelle adopté ici passe par trois phases :

découvrir l'ethnocentrisme latent chez chacun de nous ;

apprendre à distinguer ce que nous identifions consciemment comme normatif et formel et que nous pratiquons de façon subconsciente ;

apprendre aussi à découvrir nos " évidences invisibles » à " prendre des distances avec ce que nous interprétions comme naturel, quand, au fond il s'agit bien de programmes culturels que tout le monde ne partage pas » (Geneviève-Dominique de Salins citée par Louis, 2007 : 147).

L'apprenant est toujours appelé à faire un retour aux sources afin de cerner sa personnalité pour pouvoir prendre part aux interactions entre individus partageant des codes culturels différents. La culture acquise lors de l'apprentissage en langue maternelle constitue le point focal de toutes les approches développées autour de la compétence interculturelle, garante de la didactique des langues et des cultures.

Aucun enseignement des langues ne se fait sans prendre appui sur les faits culturels dont le véhicule par excellence est la langue maternelle. C'est ce dont attestent quelques-unes des recommandations de l'Assemblée parlementaire européenne :

La langue dans laquelle l'enseignement est prodigué joue un rôle essentiel dans la mesure où la maitrise de cette langue est la clé pour les processus de communication en classe et, par conséquent, pour l'acquisition des connaissances par les élèves. De nombreuses recherches ont confirmé que des formes d'enseignement fondées sur la langue maternelle augmentent significativement les chances de réussite scolaire, voire donnent de meilleurs résultats. Dans les sociétés européennes, la pratique courante de la langue officielle est la principale condition préalable à l'intégration des enfants dont la langue principale diffère de la langue officielle du pays ou de la région. De nombreuses recherches aboutissent pourtant 
au même résultat : la scolarisation immédiate de ces enfants dans une langue qu'ils maîtrisent insuffisamment, ou pas du tout, compromet sérieusement leur réussite scolaire. Un enseignement bilingue, axé sur la langue maternelle, constitue au contraire la base d'un succès à long terme. (Conseil de l'Europe, 2006)

De plus en plus, les politiques linguistiques prennent en compte l'enseignement des langues maternelles à l'école. Cette réalité est autant vraie dans les pays industrialisés que dans les pays du Tiers-Monde. Alors que pour les uns il s'agit d'améliorer le taux de réussite des enfants scolarisés par le canal de la langue maternelle, pour les autres, il est encore question d'alphabétiser l'ensemble des populations en langue maternelle, du fait de l'inaccessibilité de tous à l'école de la langue officielle.

Cette réflexion portait essentiellement sur la place de la culture en milieu institutionnel. Depuis l'adoption de la compétence interculturelle comme élément indispensable à l'enseignement/apprentissage des langues, allant de la langue maternelle à la langue étrangère, le champ épistémologique de la didactique des langues et des cultures s'engage à la rencontre des peuples, aussi divers soient-ils, tant en milieu institutionnel que lors de voyages à l'étranger pour des séjours en immersion.

L'acquisition de cette compétence nécessite la découverte de soi-même à travers un regard intérieur indispensable à la prise de conscience du regard que nous porte l'altérité, nous amenant ainsi à nous ouvrir à l'autre afin de saisir les relations que nous entretenons les uns avec les autres par l'intermédiaire de la langue. D'où la nécessité d'enseigner les langues maternelles à l'école car la langue est un patrimoine linguistique incontournable dans le dialogue qui intègre les peuples dans leurs diversités respectives.

39 Nous avons pu constater que les politiques éducatives en Afrique francophone, conscientes de la place des langues nationales à l'école, les intègrent toutes dans des textes de loi qui ne sont pas aussi effectifs sur le terrain que les langues officielles, comme le français.

40 Cependant, la compétence interculturelle, elle, est bien présente dans tout système éducatif : « En didactique des langues, à partir des années quatre-vingt et quatre-vingt-dix, le concept d'interculturalité est pris en considération dans toute pédagogie et/ou politique éducative »(Boubakour, 2010 : 18).

41 À travers l'analyse menée par Louis au sujet des différentes approches méthodologiques mises en œuvre dans l'acquisition de cette compétence, l'on a réalisé que la culture autochtone est sollicitée pour chaque pas vers l'altérité. Pour que les apprenants en classe de langue, en Afrique francophone, puissent tirer pleinement profit de cette approche, celle-ci doit intégrer leurs cultures dans leurs langues maternelles. 


\section{BIBLIOGRAPHIE}

BAPES-BAPES, Louis, « Liste officielle des manuels scolaires - Année scolaire 2010/2011 », Yaoundé, Ministère des Enseignements secondaires, 11 mai 2010. Disponible sur le site : [Http:// www.minesec.cm/uploads/manuels \%202010 \%202011/esg2010fr.pdf].

BIYA, Paul, Texte de la constitution de la République démocratique du Cameroun, Yaoundé, 18 janvier 1996. Disponible sur le site : [http://www.prc.cm/instit/consti.htm].

BOUBAKouR, Samira, « L'enseignement des langues-cultures : dimensions et perspectives », Synergies Algérie, $\mathrm{n}^{\circ}$ 9, 2012, p. 13-26. Disponible sur le site : [http://ressources-cla.univ-fcomte.fr/ gerflint/Algerie9/boubakour.pdf].

BYRAM, Michael, GRIBKOVA, Bella, et STARKEY, Hugh, Développer la dimension interculturelle de l'enseignement des langues: Une introduction pratique à l'usage des enseignants, Strasbourg, Conseil de l'Europe, 2002. Disponible sur le site : [http://www.coe.int/t/dg4/linguistic/ source/guide _ dimintercult_fr.pdf].

CHISS, Jean-Louis, « Conférence d'ouverture au Colloque », in Pagel Dario (dir.), Dialogues et cultures : Didactiques et convergences des langues et cultures, $n^{\circ}$ 51, 2006, FIPF, p. 11-16.

CollÈs, Luc, « Didactique de la littérature et diversité culturelle », in Pagel Dario (dir.), Dialogues et cultures : Didactiques et convergences des langues et cultures, $n^{\circ}$ 51, 2006, FIPF, p. 17-23.

CONSEIL DE L'EUROPE, « Recommandation 1740 : La place de la langue maternelle dans l'enseignement scolaire », Strasbourg, Conseil de l'Europe, 10 avril 2006. Disponible sur le site : [http:// assembly.coe.int/ASP/XRef/X2H-DW-XSL.asp?fileid=17421\&lang=FR].

DEFAYS, Jean-Marc, Le français langue étrangère et seconde : Enseignement et apprentissage, Sprimont (Belgique), Éditions Mardaga, 2003.

DIAGNE, Andrée-Marie, « Pour une pédagogie de l'écart et de la distanciation », in Pagel Dario (dir.), Dialogues et cultures : Didactiques et convergences des langues et cultures, $n^{\circ} 51,2006$, FIPF, p. 23-27.

GUIMATSIA, S.F., « Le bilinguisme officiel camerounais : un dangereux alibi ou une chance inouie ?», 2012. Disponible sur le site : [http://www.cameroonvoice.com/news/articlenews-6862.html].

KUITCHE FONKOU, Gabriel, Les vins aigres, Yaoundé, Éditions CLÉ, 2008.

LECLERC, Jacques, « Loi no 98/004 du 14 avril 1998 d'orientation de l'éducation au Cameroun », L'aménagement linguistique dans le monde, Québec, TLFQ, Université Laval, 27 janvier 2011. Disponible sur le site : [http://www.tlfq.ulaval.ca/axl/afrique/cameroun.htm].

LOUIS, Vincent, Interactions verbales et communication interculturelle en FLE : De la civilisation française à la compétence (inter)culturelle, Cortil-Wodon (Belgique), Éditions modulaires européennes, Collection « IRIS », 2007.

NOLA, Bienvenu, «Quelle pédagogie pour l'enseignement du français dans le contexte multilingue en Afrique? ", in Pagel Dario (dir.), Dialogues et cultures : Didactiques et convergences des langues et cultures, $n^{\circ}$ 51, 2006, FIPF, p. 125-128. 
ORGANISATION INTERNATIONALE DE LA FRANCOPHONIE, « ÉLAN-Afrique : une nouvelle offre francophone en matière d'enseignement bilingue pour mieux réussir à l'école », Paris, 8 septembre 2011. Disponible sur le site : [http://www.francophonie.org/IMG/pdf/CP2511_ELAN Afrique.pdf].

WOLFF, Alexandre et Gonthier, Josiane, La langue française dans le monde 2010, Paris, Nathan, 2010.

\section{RÉSUMÉS}

Cet article est une réflexion sur la place de la culture et des langues maternelles, donc de l'identité culturelle, dans l'enseignement des langues, en Afrique francophone contemporaine. Depuis la prise en compte de la compétence interculturelle dans l'acquisition des langues, on est passé de la didactique « des langues » à la didactique " des langues et cultures », confirmant ainsi l'importance de la culture, donc de l'identité culturelle de chaque individu, en la classe de langue. Cependant, ce regard nouveau sur les relations entre la langue et la culture dans l'enseignement n'est pas une question réellement prise en compte dans les systèmes éducatifs de tous les pays de l'Afrique francophone. Aujourd'hui encore, dans certains des pays africains, enseignants et apprenants utilisent la langue française pour l'étude de leurs cultures. C'est cette question que nous aborderons à travers la notion de «compétence interculturelle » dans les systèmes d'enseignement/apprentissage des langues et cultures en Afrique francophone.

By the early 80s, issues concerning the education of immigrant children in Europe resulted in the adoption of intercultural competence within the Council of Europe. Language theorists switched from the didactics of "languages" to the didactics of "languages and cultures", hence emphasising the importance of culture and, hence, the cultural identity of each individual in foreign language classes. This new vision on the relationship between language and culture is almost non-existent in French-speaking Africa. Today, teachers and learners alike use the French language to study their numerous African cultures. With the intercultural approach, any language learner is required to move away from the old practice in order to partake in the ongoing "dialogue with people" from diverse backgrounds; since they will have observed and understood their values and cultures while preserving theirs. The teaching and learning of African languages and cultures being formally absent in schools raises the following question: how does intercultural training take place in this context?

\section{INDEX}

Keywords : intercultural competence, culture, french-speaking, African Countries, mother tongue

Mots-clés : compétence interculturelle, culture, Afrique francophone, langue maternelle, langue française

\section{AUTEUR}

\section{CHRISTIANE MOUTO BETOKO}

Université de Liège 